\title{
Separation of Two-Electron Photoexcited Atomic Processes near the Inner-Shell Threshold
}

\author{
M. Kavčič, ${ }^{1}$ M. Žitnik, ${ }^{1}$ K. Bučar, ${ }^{1}$ A. Mihelič, ${ }^{1}$ M. Štuhec, ${ }^{1}$ J. Szlachetko, ${ }^{2,3}$ W. Cao, ${ }^{2}$ R. Alonso Mori, ${ }^{4}$ and P. Glatzel ${ }^{4}$ \\ ${ }^{1}$ J. Stefan Institute, Jamova 39, SI-1000, Ljubljana, Slovenia \\ ${ }^{2}$ Physics Department, University of Fribourg, CH-1700 Fribourg, Switzerland \\ ${ }^{3}$ Jan Kochanowski University, Institute of Physics, Kielce, Poland \\ ${ }^{4}$ European Synchrotron Radiation Facilty (ESRF), Grenoble, France
}

\begin{abstract}
By means of a high resolution resonant inelastic x-ray scattering spectroscopy, we have for the first time separated spectral features pertaining to different two-electron atomic processes in the vicinity of an innershell threshold. Contributions of shakeoff, shakeup, and resonant $1 s 3 p$ double excitations were extracted from the Ar $K M-M_{2,3} M$ x-ray satellite line intensity measured as a function of photon energy from [1s $\left.3 p\right]$ double excitation threshold to saturation. The isolated $[1 s 3 p] n l^{\prime} l^{\prime}$ excitation spectrum is critically compared to the outcome of the multiconfiguration Dirac-Fock model with relaxation.
\end{abstract}

According to the nature of photon-electron interaction a photon is absorbed only by a single electron. On the other hand, photoionization of an inner-shell electron is sometimes accompanied by removal of another electron (shakeoff) or its excitation into a higher empty atomic level (shakeup), and below the double photoionization threshold, doubly excited atomic states can be created. These multielectron excitations are straightforward manifestations of the breakdown of the independent electron picture as they appear due to the electron-electron interactions. Despite the persistent and intensive research the mechanisms of electron-electron correlations have not been fully elucidated yet. While the well-known shake mechanism (governed by monopole transitions between relaxed states) is commonly taken as a synonym for the double photoionization or excitation process, the presence of dynamical effects in the near-threshold region makes the interpretation of double photoexcitation spectra much more complicated.

The main experimental problem hindering the study of near-threshold multielectron excitations is the overlap of the shakeoff, shakeup, and double excitation spectral contributions. Strong dependence of the shakeoff to shakeup probability ratio on the atomic number and atomic shell was employed to bypass this difficulty in some special cases. While the shakeup mechanism fully dominates the electron excitation following $1 s$ photoionization of Be [1], the shakeoff prevails in the $1 s 2 p$ double photoionization of mid- $Z$ atoms [2]. It was found that a time-dependent perturbation theory [3] describes well the double photoionization of outer-shell electrons in low- $Z$ atoms; for inner shells and higher $Z$ atoms significant deviations were found $[4,5]$, indicating that other mechanisms besides the shakeoff are at work. In the latest work a semiclassical knockout effect was suggested to dominate the near-threshold double $1 s$ photoionization in $3 d$ transition metals [6].

One of the most interesting multielectron features is double excitations exhibiting discrete resonances in the preedge region. These processes are extremely weak when both electrons are excited from core levels, but they become more probable if a valence electron takes part in the excitation. In such cases structural solid-state effects obscure spectral details related to multiple atomic excitations, limiting such studies to gaseous atomic targets. This is the main reason why up to now multiple atomic excitations involving inner-shell electron were studied only by absorption spectroscopy. Since they are relatively easy to manipulate experimentally, and handle theoretically, noble gas atoms have been investigated in detail [7-11]. An inherent problem of absorption studies remains proper separation of the dominating single-electron photoionization component underlying a weak multielectron signal. The related experimental uncertainty can partially explain why only seldom attempts were made to extend the calculations of energy levels and interpret the strength of multielectron excitations $[12,13]$.

The multielectron signal was effectively separated from the dominating single-electron contribution in the pioneering work of Armen et al. [14]. They recorded a series of Auger satellite spectra with energy splitting large enough even to allow for an approximate separation of the shakeup and shakeoff lines in case of $\operatorname{Ar} 1 s 3 p$ photoionization. Although relatively strong satellite intensity measured at $3225 \mathrm{eV}$ indicated the transition to $[1 s 3 p] 4 p^{2}$ doubly excited states, the density of sampling points in the nearthreshold region was not high enough to extract double excitations.

In this Letter we report the first complete separation of spectral features pertaining to different multielectron processes by using a high resolution resonant inelastic x-ray scattering (RIXS) spectroscopy. The characteristic nearthreshold energy dependency of $\operatorname{Ar}[1 s 3 p]$ photoexcitation allowed an isolation of separate multielectron contributions, despite that it is virtually impossible to resolve them in an isolated $\mathrm{x}$-ray spectrum. The extracted double $[1 s 3 p]$ excitation spectrum was compared with detailed 
calculations which include correlations in the ground and excited atomic states and account for the relaxation effects. The presented approach could be applied to other low density targets such as metallic vapors (involving the open shell in the initial state), molecular targets (involving competitive decay channels, elaborated basis sets, and structural effects), or gaseous targets in the external fields, which may provide data to stimulate advanced theoretical approaches in the future.

Measurements were carried out at the ID26 beam line of the European Synchrotron Radiation Facility. The incident photon energy was tuned by means of a cryogenically cooled double $\mathrm{Si}(111)$ crystal monochromator with a band pass of $\sim 0.5 \mathrm{eV}$ at $3.2 \mathrm{keV}$. The incident flux on the target was $5 \times 10^{12}$ photons/ sec. Higher harmonics were suppressed by two Si mirrors at $5.5 \mathrm{mrad}$ and $6 \mathrm{mrad}$, respectively. The incident beam with cross section 50 (height) $\times 250$ (width) $\mu \mathrm{m}^{2}$ was impinging on a stainless steel cylindrical target cell filled with 500 mbar of $\mathrm{Ar}$ and separated from the spectrometer vacuum chamber with $12.5 \mu \mathrm{m}$ thick Kapton windows. The target $K M_{2,3} \mathrm{x}$-ray fluorescence spectra were measured using a crystal spectrometer in Johansson geometry with a $500 \mathrm{~mm}$ Rowland circle radius. The first order reflection of a $\mathrm{Si}(111)$ crystal was used, and the diffracted photons were detected with a thermoelectrically cooled $\left(-40^{\circ} \mathrm{C}\right) \mathrm{CCD}$ camera with $22.5 \times 22.5 \mu \mathrm{m}^{2}$ pixel size. The overall energy resolution of the spectrometer was $\sim 0.6 \mathrm{eV}$ at the energy of the $\mathrm{Ar}$ $K \beta$ line. The detection angle was perpendicular to the horizontally polarized incident photon beam to reduce the background due to elastic scattering. In order to use the large target interaction volume and at the same time exploit the length of the CCD camera to simultaneously measure the whole $K M_{2,3}$ spectrum at fixed detector position, the target was placed well inside the Rowland circle at a distance of $27 \mathrm{~cm}$ in front of the diffraction crystal. A series of $81 \mathrm{x}$-ray emission spectra was recorded starting at an incident beam energy of $3219 \mathrm{eV}$ and than changing the energy in steps of $0.25 \mathrm{eV}$ up to $3239 \mathrm{eV}$. Seventeen more spectra were collected between 3240 and $3300 \mathrm{eV}$ to determine a rather slow energy evolution of the shakeoff component. All spectra were normalized to the photon dose deduced from the photon current density measured before spectrometer entrance. The acquisition time for each spectrum was 250 seconds. Since the absorption coefficient is not changing significantly over the measured energy interval, the estimated self-absorption correction was less than 3\% and was neglected in our analysis.

Figure 1 shows a $K M_{2,3}$ spectrum measured at three different incident photon energies near the $1 s 3 p$ threshold. By correlating the measured excitation energy dependence of the satellite intensity with multielectron features identified in the absorption spectrum, Deslattes et al. [7] attributed the satellite line in the presented $\operatorname{Ar} K M_{2,3}$ spectrum to the $1 s 3 p$ double vacancy state. This is confirmed also by

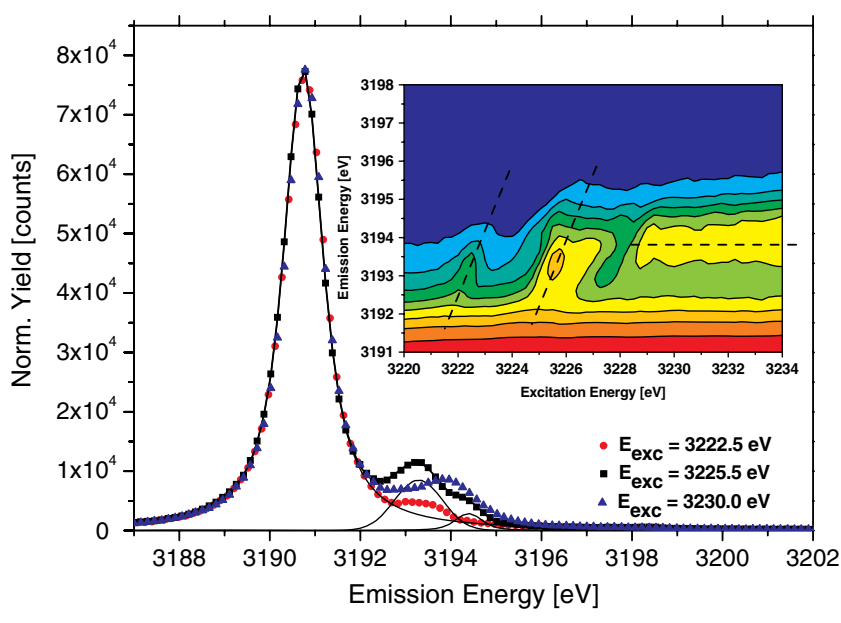

FIG. 1 (color online). High resolution $\mathrm{Ar} K M_{2,3}$ x-ray emission spectrum measured at three excitation energies close to the $1 s 3 p$ threshold. The full line corresponds to the fit and its components for the spectrum measured at $3225.5 \mathrm{eV}$. A contour plot in the inset represents a full scan of the satellite line over the $1 s 3 p$ near-threshold region.

the configuration-interaction calculations of $\mathrm{Ar} K M_{2,3}$ emission spectra performed by Dyall and Grant [15]. From Fig. 1 it is evident that near the $1 s 3 p$ threshold the intensity as well as the line shape of the satellite line strongly depend on the excitation energy, which is typical for the resonant scattering [16,17]. Since both energies (excitation and emission) enter the RIXS cross section, it is customary to present data by a $2 \mathrm{D}$ contour map which represents a RIXS spectral plane. The inset of Fig. 1 presents such a plane for the $\operatorname{Ar} K M-M_{2,3} M$ satellite line in the $1 s 3 p$ near-threshold region. The most outstanding feature of this map is the clear separation of two resonant excitations and the following edge. A linear Raman-Stokes dispersion is observed for the first two contributions, which are evolving along diagonal lines on the contour plot. A sharp edge typical for the shakeup process follows the region of doubly excited resonances. In contrast with two resonant contributions, no excitation energy dependence is observed after the edge, an indication of the excitation into continuum. In the RIXS spectral plane the character of different multielectron processes is clearly manifested.

In order to extract the $K M-M_{2,3} M$ satellite line intensity evolution, each measured and normalized $K M_{2,3}$ x-ray spectrum was analyzed by a least square fitting procedure. A single Voigt profile was employed to fit the diagram line, while two Voigt profiles were needed to obtain a reasonably good fit of the $K M-M_{2,3} M$ satellite line (Fig. 1). The $1 s 3 p$ double photoexcitation spectrum is given by plotting integral counts of the extracted satellite contribution versus the excitation energy (Fig. 2). The overall shape of the excitation curve resembles roughly the shape reported by Deslattes et al. (Fig. 9 of Ref. [7]), but the very high density of points in the near-threshold region reveals much more 


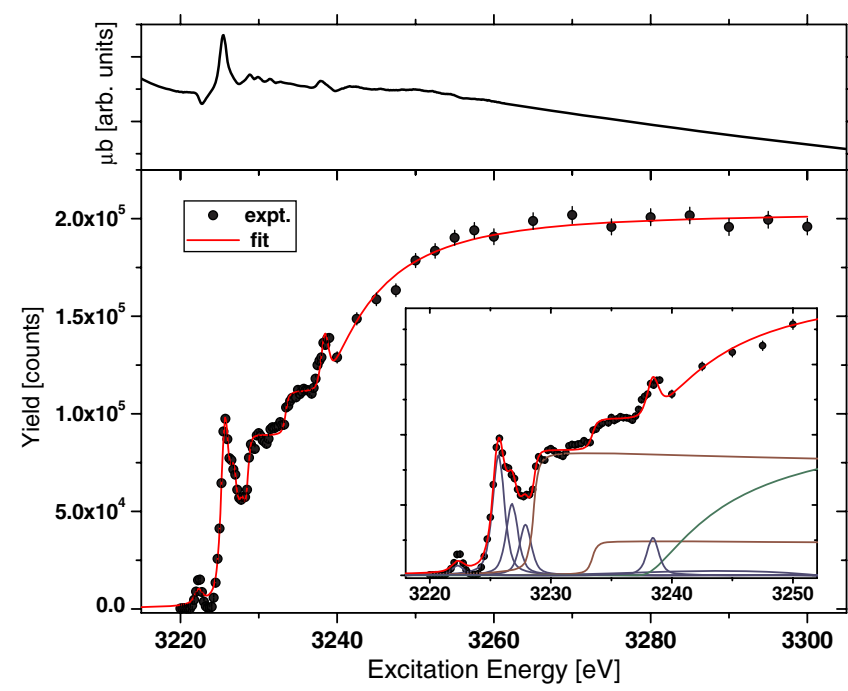

FIG. 2 (color online). Intensity evolution of the $K M-M_{2,3} M$ satellite line as a function of the excitation energy. The solid curve represents the fit of the model presented in the text. In the inset the enlarged view of the near-threshold region is presented exhibiting main resonances, two shakeup edges, and a smooth shakeoff contribution. For comparison the corresponding detail of the absorption spectrum is presented above.

detail, which are of main interest here. The excitation spectrum was modeled using the following components: Voigt peaks for resonant double excitations, linear combination of Lorentzian and Gaussian cumulative distributions for shakeup channels, and exponential saturation functional form given by Thomas [3] for the shakeoff channel. The last two were both additionally weighted by a slowly decreasing exponential factor given experimentally from the excitation energy dependence of the $K M_{2,3}$ diagram line intensity. The Lorentzian width of the Voigt peaks was fixed to $0.69 \mathrm{eV}$ according to the $\Gamma_{1 s}$ experimental value given by Morgan, Bartlett, and Sagurton [18]. The Gaussian width of the Voigt and shakeup components was a free fit parameter yielding a value of $0.75 \mathrm{eV}$. According to the $Z+1$ model, the shake energy in the Thomas model was fixed to $17.9 \mathrm{eV}$, the value obtained from the $3 p$ binding energy of potassium [19]. With these components we could reproduce the overall profile of the photoexcitation spectrum (Fig. 2). The results show that in the asymptotic limit the shakeoff channel prevails over the shakeup. This is consistent with the previous result of Armen [14] and is in contrast with the theoretical prediction by Dyall [20]. The $[1 s 3 p]$ shakeoff threshold energy was found at $3236.3 \pm 0.7 \mathrm{eV}$ and $[1 s 3 p] 4 p ; 5 p$ shakeup edges at $3228.56 \pm 0.04 \mathrm{eV}$ and $3233.43 \pm 0.08 \mathrm{eV}$, respectively. These values are consistent with the HartreeFock calculated average energies given by Deutsch, Maskil, and Drube [9].

The resonant part of the $[1 s 3 p]$ spectrum was separated from the excitation profile in Fig. 2 by subtracting the shakeup and shakeoff contributions. The resonant $\mathrm{x}$-ray yield was then converted into the corresponding absolute $[1 s 3 p] n \ln ^{\prime} l^{\prime}$ excitation cross section, which is presented in Fig. 3. The scaling factor was obtained by comparing the total measured $K M$ x-ray yield at given excitation energy with corresponding $1 \mathrm{~s}$ cross section extracted from the absolute measurement of the total photoabsorption cross section by Deutsch, Maskil, and Drube [9]. In this procedure, the measured satellite emission yield was additionally multiplied by a factor 1.34 , a ratio of calculated oscillator strengths for $[1 s] \rightarrow[3 p] /[1 s 3 p] \rightarrow\left[3 p^{2}\right]$ radiative transitions. Ten emission spectra measured at highest excitation energies (3260-3300 eV) were used to determine the scaling factor.

A comparison of our experimental resonant $[1 s 3 p]$ excitation spectrum with the curve, reconstructed from the only existing theoretical data of Sukhorukov et al. [12], is presented in Fig. 3. After an energy shift of $1.3 \mathrm{eV}$, needed to match the positions of maximum intensities, a good agreement is noted except in the region of the lowest $[1 s 3 p] 4 s^{2}$ resonance, which was not included in the calculation [12].

We have built the theoretical double excitation spectrum by calculating the dipole oscillator strengths into $[1 s 3 p] n \ln ^{\prime} l^{\prime}$ excited atomic states, taking a full account of atomic relaxation (Table I). The states were determined by the Dirac-Fock self-consistent field scheme [21], while the nonorthogonality was handled by the nonrelativistic code of Zatsarinny [22]. To represent excited states, the ${ }^{1} P$ terms of eight configurations were mixed in the average

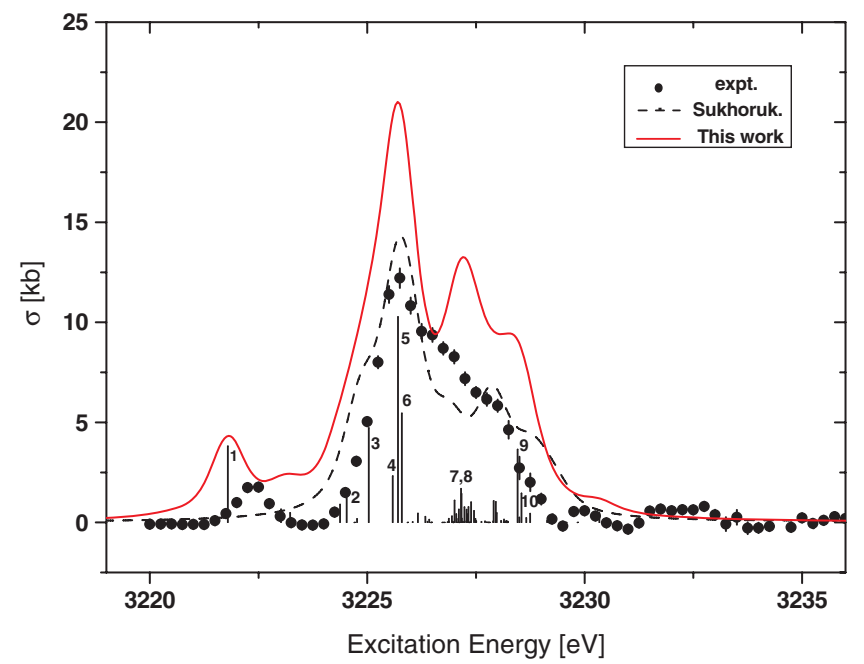

FIG. 3 (color online). Resonant part of the measured [1s3p] excitation spectrum compared with our multiconfiguration Dirac-Fock calculated spectrum and the theoretical spectrum by Sukhorukov et al. [12]. Both theoretical spectra were built from the calculated stick spectrum using Voigt profiles with $0.69 \mathrm{eV}$ Lorentzian width and $0.5 \mathrm{eV}$ Gaussian width according to the $\Gamma_{1 s}$ natural line width and resolution of the beam line monochromator, respectively. The theoretical spectrum by Sukhorukov et al. has been shifted by $+1.3 \mathrm{eV}$. 
TABLE I. Calculated energies, oscillator strengths, and weights for two dominant configurations in the $[1 s 3 p]$ final state atomic functions for the main components marked in Fig. 3.

\begin{tabular}{rlll}
\hline \hline$N$ & $E[\mathrm{eV}]$ & $f \times 10^{4}$ & \\
\hline 1 & 3221.80 & 0.473 & $0.894 s^{2} / 0.094 p^{2}$ \\
2 & 3224.53 & 0.208 & $0.684 p^{2} / 0.193 d^{2}$ \\
3 & 3225.04 & 0.586 & $0.394 s 4 d / 0.244 p^{2}$ \\
4 & 3225.59 & 0.288 & $0.734 s 4 d / 0.134 p^{2}$ \\
5 & 3225.71 & 1.274 & $0.584 s 4 d / 0.254 p^{2}$ \\
6 & 3225.80 & 0.676 & $0.484 s 4 d / 0.224 p^{2}$ \\
7 & 3227.16 & 0.209 & $0.544 p 5 p / 0.373 d 4 d$ \\
8 & 3227.17 & 0.178 & $0.473 d 4 d / 0.424 p 5 p$ \\
9 & 3228.46 & 0.452 & $0.523 d 4 d / 0.374 p 5 p$ \\
10 & 3228.55 & 0.181 & $0.823 d 4 d / 0.114 p 5 p$ \\
\hline \hline
\end{tabular}

level mode: $4 p^{2}, 3 d^{2}, 4 s^{2}, 3 d 4 s, 4 p 5 p, 4 s 4 d, 3 d 4 d$, and $4 d^{2}$. Apart from obtaining good agreement of calculated energies without any energy shift, our calculations also nearly reproduced the peak pertaining to $[1 s 3 p] 4 s^{2}$ resonance. Further optimized level studies showed that its relative energy difference to the main peak has diminished, coming in closer agreement with experiment. As suggested already by Deslattes et al. [7], our results confirm that the first peak, observed at $3222.3 \mathrm{eV}$ excitation energy, originates from the conjugate shakeup amplitude (symbolically denoted by $\langle 1 s \mid 4 s\rangle\langle 3 p|r| 4 s\rangle$ ), mainly on account of the surprisingly large dipole matrix element between $3 p$ and $4 s$ atomic orbitals. It is to be noted that the $[1 s 3 p] 4 s^{2}$ signal in the absorption spectrum is effectively dispersed due to the Fano interference while in the fluorescence mode this effect is entirely absent, allowing more direct comparison with theory.

Our calculations represent a step forward, but it seems that comparison on the absolute scale fails with respect to the old calculations. Here it is necessary to note that while the $\mathrm{x}$-ray emission from an ionized atom is practically isotropic, especially far above the threshold, allowing direct comparison with a corresponding excitation cross section, this in principle does not hold for the fluorescence angular pattern emitted by excited atomic states with well defined total angular momentum [23]. In fact, the presented experimental spectrum is proportional to $(d \sigma / d \Omega)_{\|}$, the differential resonant inelastic x-ray scattering cross section taken along the incident polarization axis and integrated over the $K M$ emission energy range. While there is no ambiguity in the measured shape since in our geometry both polarization components give exactly the same spectral shapes, the absolute values might be suppressed due to the fact that in the direction of incident polarization the resonant $J=1 \rightarrow J=0$ contribution is absent. Together with partial $K M$ fluorescence rates, which could depend on the initial resonant state, this may be the main source of remaining discrepancies between measured and calculated values.

In conclusion, a comprehensive RIXS study of the gas phase target is presented for the case of Ar $1 s 3 p$ double excitation. Different multielectron contributions are clearly resolved, and detailed evolution of $K M-M_{2,3} M$ satellite intensity near the threshold is presented. The state-of-the-art multiconfiguration Dirac-Fock simulation favorably compares to the resonant $\operatorname{Ar} 1 s 3 p$ double excitation spectrum extracted from the experimental data. This Letter demonstrates the capability of RIXS spectroscopy to perform high resolution studies of diluted targets in a due time. The possibilities are opened for further challenging experiments on other atomic and molecular targets.

This work was supported by the Slovenian Ministry of Higher Education, Science, and Technology (research program P1-0112) and by the Swiss National Science Foundation.

[1] M. O. Krause and C.D. Caldwell, Phys. Rev. Lett. 59, 2736 (1987).

[2] M. Deutsch, O. Gang, K. Hämäläinen, and C. C. Kao, Phys. Rev. Lett. 76, 2424 (1996).

[3] T. D. Thomas, Phys. Rev. Lett. 52, 417 (1984).

[4] R. Diamant et al., Phys. Rev. Lett. 91, 193001 (2003).

[5] E. P. Kanter et al., Phys. Rev. A 73, 022708 (2006).

[6] S. Huotari et al., Phys. Rev. Lett. 101, 043001 (2008).

[7] R. D. Deslattes, R. E. LaVilla, P. L. Cowan, and A. Henins, Phys. Rev. A 27, 923 (1983).

[8] K. G. Dyall and R.E. LaVilla, Phys. Rev. A 34, 5123 (1986).

[9] M. Deutsch, N. Maskil, and W. Drube, Phys. Rev. A 46, 3963 (1992).

[10] S. J. Schaphorst et al., Phys. Rev. A 47, 1953 (1993).

[11] I. Arčon, A. Kodre, M. Štuhec, D. Glavič-Cindro, and W. Drube, Phys. Rev. A 51, 147 (1995).

[12] V.L. Sukhorukov, A. N. Hopersky, I. D. Petrov, V. A. Yavna, and V.F. Demkhin, J. Phys. (Paris) 48, 1677 (1987).

[13] H. P. Saha, Phys. Rev. A 42, 6507 (1990).

[14] G. B. Armen et al., Phys. Rev. Lett. 54, 182 (1985).

[15] K. G. Dyall and I. P. Grant, J. Phys. B 17, 1281 (1984).

[16] A. Kotani and S. Shin, Rev. Mod. Phys. 73, 203 (2001).

[17] F. Gel'mukhanov and H. Ågren, Phys. Rep. 312, 87 (1999).

[18] D. V. Morgan, R. J. Bartlett, and M. Sagurton, Phys. Rev. A 51, 2939 (1995).

[19] K. D. Sevier, At. Data Nucl. Data Tables 24, 323 (1979).

[20] K. G. Dyall, J. Phys. B 16, 3137 (1983).

[21] K. G. Dyall, I. P. Grant, C. T. Johnson, F. A. Parpia, and E. P. Plummer, Comput. Phys. Commun. 55, 425 (1989).

[22] O. Zatsarinny, Comput. Phys. Commun. 98, 235 (1996).

[23] S. H. Southworth, D. W. Lindle, R. Mayer, and P. L. Cowan, Phys. Rev. Lett. 67, 1098 (1991). 